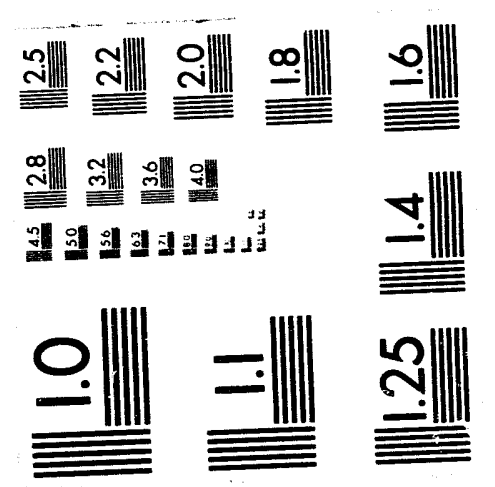



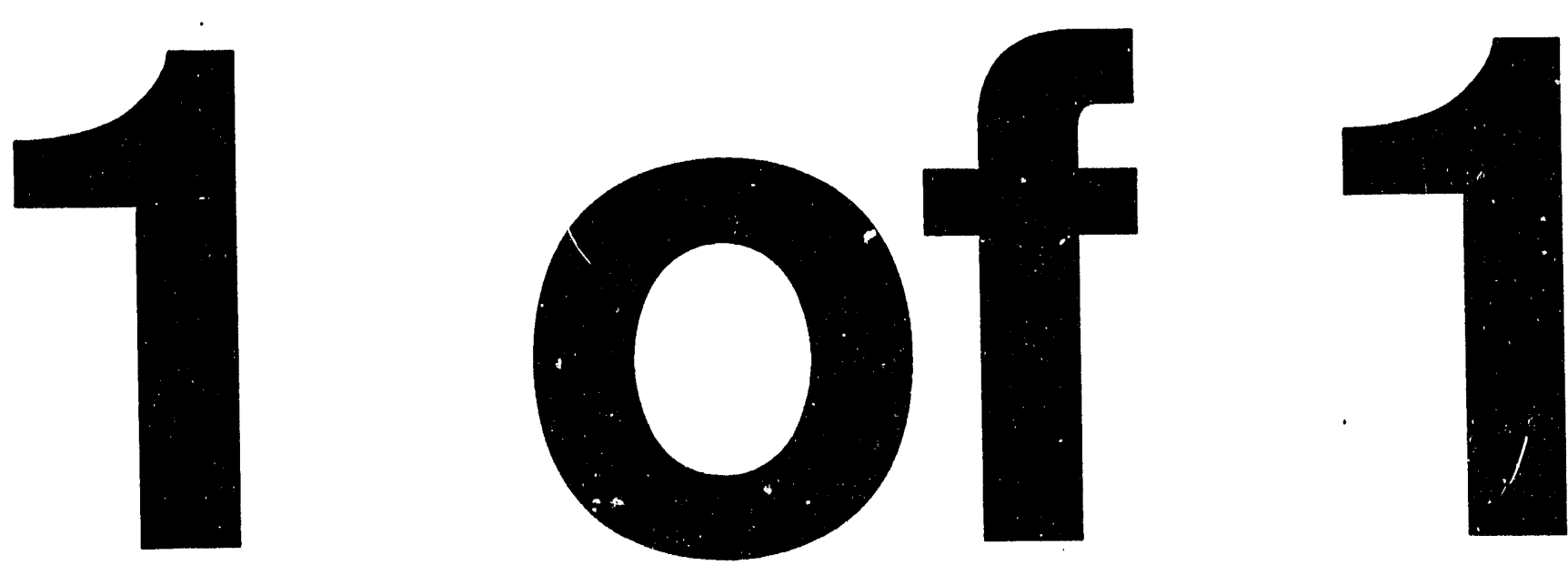


\section{FUSION STUDIES OSTI \\ APR 11 994}

DE-FG05-80ET-53088-648

IFSR \#648

Decay of Magnetic Helicity Producing Polarized Alfvén Waves

Z. YOSHIDA

Faculty of Engineering

University of Tokyo

Hongo, Tokyo 113, Japan

and

S.M. MAHAJAN

Institute for Fusion Studies

The University of Texas at Austin

Austin, Texas 78712

February 1994

THE UNIVERSITY OF TEXAS

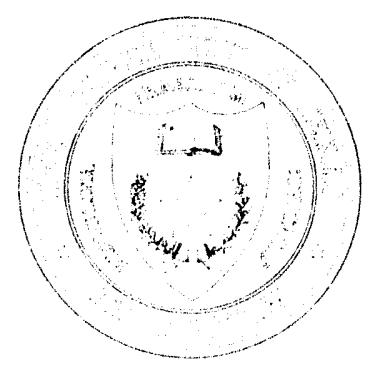

AUSTIN 


\title{
Decay of Magnetic Helicity Producing Polarized Alfvén Waves
}

\author{
Z. YOSHIDA \\ Faculty of Engineering \\ University of Tokyo \\ Hongo, Tokyo 113, Japan \\ and \\ S.M. MAHAJAN \\ Institute for Fusion Studies \\ The University of Texas at Austin \\ Austin, Texas 78712
}

\begin{abstract}
When a super-Alfvénic electron beam propagates along an ambient magnetic field, the left-hand circularly polarized Alfvén wave is Cherenkov-emitted (two stream instability). This instability results in a spontaneous conversion of the background plasma helicity to the wave helicity. The background helicity induces a frequency (energy) shift in the eigenmodes, which changes the critical velocity for Cherenkov emission, and it becomes possible for a sub-Alfvénic electron beam to excite a nonsinguiar Alfvén mode.
\end{abstract}

PACS Numbers: $52.35 . \mathrm{Bj}, 41.60 . \mathrm{Bq}, 52.35 . \mathrm{Qz}, 42.25 . \mathrm{Ja}$

\section{DISCLAIMER}

This report was prepared as an account of work sponsored by an agency of the United States Government. Neither the United States Gnvernment nor any agency thereof, nor any of their employees, makes any warranty, express or implied, or assumes any legal liability or responsibility for the accuracy, completeness, or usefulness of any information, apparatus, product, or process disclosed, or represents that its use would not infringe privately owned rights. Reference herein to any specific commercial product, process, or service by trade name, trademark, manufacturer, or otherwise does not necessarily constitute or imply its endorsement, recommendation, or favoring by the United States Government or any agency thereof. The views and opinions of authors expressed herein do not necessarily state or reflect those of the United States Government or any agency thereof. 
Damping of a circularly polarized Alfvén wave generates a current in the background plasma. ${ }^{1,2}$ This phenomenon can be interpreted as helicity transfer from the Alfvén wave to the background plasma. The helicity absorption is equivalent to a change in the twist of the ambient magnetic field, and hence to the production of a mean current.

In this Letter we consider the inverse process, i.e. a decay of the mean-field helicity producing circularly polarized Alfvén waves. A fast electron stream carrying a net current ${ }^{3}$ will be the free energy source for this spontaneous process to occur. It is well known that super-Alfvénic beam electrons (beam velocity $v_{b}>$ Alfvén velocity $v_{A}$ ) may Cherenkovemit Alfvén waves, which are left-hand circularly polarized. ${ }^{4}$ By considering the balance between the wave and the background helicity, we show that this 'two-stream instability' is a spontaneous process producing wave helicity. The excited waves have wavenumber anisotropy reflecting the polarity of the background helicity. ${ }^{5}$ An interesting effect of the helicity appears in determining the critical velocity of the beam. The background helicity yields a frequency (energy) shift in the eigenmodes, and, when a nonsingular eigenmode occurs, it can be exited by a sub-Alfvénic electron beam.

Let us start with reviewing the local dispersion relation of the two-stream system. We consider a cold electron beam of velocity $\mathbf{v}_{b}$ propagating parallel to the ambient uniform magnetic field $\mathbf{B}_{0}$, and carrying a current $\mathbf{j}_{b}=-e n_{b} \mathbf{v}_{b}$, where $e$ is the elementary charge and $n_{b}$ is the density of the beam electrons. The background electrons and ions (singly charged and density $=n_{0}$ ) are cold with zero mean velocity. The transverse wave propagating parallel to $\mathbf{B}_{0}$ is described by the vector potential $\widetilde{\mathbf{A}}_{\perp}$, and the wave vector $k_{\|} \mathbf{b}$, where $\mathbf{b}=\mathbf{B}_{0} / B_{0}$ and $\perp$ is perpendicular to $\mathbf{B}_{0}$. The left-hand circularly polarized wave (L-wave) is characterized by the polarization relation $\widetilde{\mathbf{A}}_{\perp} \times \widetilde{\mathbf{A}}_{\perp}^{*}=i\left|\widetilde{\mathbf{A}}_{\perp}\right|^{2} \mathbf{b}$. We consider a long wave length $\left(\left|k_{\|}\right| \ll \omega_{c e} / v_{b}\right)$ and low frequency $\left(|\omega| \ll \omega_{c i}\right)$, where $\omega_{c e}\left(\omega_{c i}\right)$ is the electron (ion) 
cyclotron frequency. The dispersion relation for the L-wave reads $\left[\delta=n_{b} / n_{0}, \alpha=\delta \omega_{c i} / v_{A}^{2}\right]$

$$
\left(\frac{\omega}{v_{A}}\right)^{2}-k_{\|}^{2}-\alpha\left(\omega-k_{\|} v_{b}\right)=0
$$

with the solution ${ }^{4}$

$$
\frac{\omega}{\omega_{c i}}=\frac{\delta}{2}\left[1 \pm \sqrt{1-\left(\frac{v_{b}}{v_{A}}\right)^{2}+\left(\frac{2 k_{\|} v_{A}}{\delta \omega_{c i}}-\frac{v_{b}}{j_{A}}\right)^{2}}\right] .
$$

When $v_{b}>v_{A}$, instability (complex $\omega$ ) occurs ir the range of $k_{\|} \approx \delta v_{b} \omega_{c i} / 2 v_{A}^{2}$. This instability of the L-wave is induced by the coupling with a negative energy whistler wave (right-hand circularly polarized wave) which propagates backward on the beam electrons. Therefore, a positive energy L-wave is Cherenkov-emitted on the background ions. The L-wave propagates in the direction of the electron beam, i.e. $k_{\|}$and $v_{b}$ have the same sense.

Let us now compare the wave helicity and the background helicity. The wave helicity density is given by $\left[\widetilde{\mathbf{B}}=i k_{\|} \mathbf{b} \times \widetilde{\mathbf{A}}\right]$

$$
h_{L}=\left\langle\widetilde{\mathbf{A}}_{\perp} \cdot \widetilde{\mathbf{B}}\right\rangle=-k_{\|}\left|\widetilde{\mathbf{A}}_{\perp}\right|^{2}
$$

where $\langle\cdot\rangle$ represents the time average. The background helicity is generated by a mean current $\mathbf{J}_{0}$, which consists of the beam current $\mathbf{j}_{b}$ and the bulk plasma current $j_{0}$. To keep the discussion simple, we neglect $\mathbf{j}_{0}$ here, but shall keep it later when we include nonuniformity of $\mathbf{B}_{0}$ in our analysis. Since $\mathbf{J}_{0}=\nabla \times \mathbf{B}_{0} / \mu_{0}$ is approximately parallel to $\mathbf{B}_{0}$ in a low-beta plasma, we may write

$$
\nabla \times \mathbf{B}_{0}=\lambda \mathbf{B}_{0}
$$

where $\lambda$ is a real scalar function. Approximating $\lambda$ be a constant in the neighborhood of the beam, we may write the vector potential $\mathbf{A}_{0}=\mathbf{B}_{0} / \lambda$ in the Coulomb gauge, which is consistent to the expression of $\widetilde{\mathbf{A}}_{\perp}$ in the transverse wave. The background helicity is given by

$$
h_{0}=\mathbf{A}_{0} \cdot \mathbf{B}_{0}=\lambda\left|\mathbf{A}_{0}\right|^{2} .
$$


Since $k_{\|}\left(\approx \delta v_{b} \omega_{c i} / 2 v_{A}^{2}\right)$ and $\lambda\left(=\mu_{0} \mathbf{j}_{b} \cdot \mathrm{B}_{0} / B_{0}^{2}=-\mu_{0} e n_{b} v_{b} / B_{0}\right)$ have different signs, it turns out that the helicity density $h_{L}$ of the produced L-wave has the same sense of the background (beam) helicity density $h_{0}{ }^{6}$

In the local dispersion relation (1) the energy balance among the beam, the Alfvén wave, and the backward whistler wave is displayed, while the effect of the background helicity (i.e. the nonuniformity of $\mathbf{B}_{0}$ ) is omitted. We now extend our analysis to a nonuniform plasma so that interesting effect associated with the background helicity can be delineated.

For low frequency $\left(|\omega| \ll \omega_{c i}\right)$ incompressible dynamics of a low-beta plasma, it is adequate to keep only the parallel component of the vector potential, and write the electric field $\widetilde{\mathbf{E}}=-\left(\partial_{t} A_{\|}\right) \mathrm{b}-\nabla \phi$. The defining equations are

$$
\tilde{E}_{\|}=i \omega A_{\|}-\nabla_{\|} \phi=0,
$$

the charge neutrality condition

$$
\nabla_{\|} j_{\|}+\nabla_{\perp} \cdot \dot{j}_{\perp}=0
$$

and the parallel Ampére's law

$$
j_{\|}=-\mu_{0}^{-1} \nabla_{\perp}^{2} A_{\|},
$$

where $j_{\|}$and $j_{\perp}$ represents, respectively, the parallel and perpendicular components of the current density, $\nabla_{\|}=\mathbf{b} \cdot \nabla$, and $\nabla_{\perp}=\nabla-\nabla_{\|}$. The perpendicular current $\mathbf{j}_{\perp}$ is dominated by the ion polarization current

$$
\mathrm{j}_{p}=\frac{i \omega \nabla_{\perp} \phi}{\mu_{0} v_{A}^{2}}
$$

together with the perpendicular electron current $j_{w}$ which is induced by the whistler wave propagating on the beam electrons. We may use the local conductivity tensor and neglect the Faraday rotation effect, and obtain $\mathbf{j}_{w}$ induced by the L-polarized transverse electric field $\left(-\nabla_{\perp} \phi\right)$

$$
\mathrm{j}_{w}=\frac{\alpha}{i \omega}\left(\omega-k_{\|} v_{b}\right) \nabla_{\perp} \phi
$$


Combining (6)-(10) yields the eigenmode equation

$$
\nabla_{\|}\left[\nabla_{\perp}^{2}\left(\nabla_{\|} \phi\right)\right]+\nabla_{\perp} \cdot\left[\left(\frac{\omega^{2}}{v_{A}^{2}}-\alpha\left(\omega-k_{\|} v_{b}\right)\right) \nabla_{\perp} \phi\right]=0 .
$$

Let us write $\phi(r, \theta, \varphi)=\sum_{m, n} \phi_{m, n}(r) \exp i(-m \theta+n \varphi-\omega t)$, where $r$ is the radial coordinate, $\theta$ and $\varphi$ are the poloidal and toroidal angles, respectively. We assume a large aspect ratio (major radius $R \gg r$ ), and neglect the toroidal coupling of different Fourier modes. Then, (11) is approximated by

$$
\frac{d}{r d r}\left(f\left(\omega, k_{\|}\right) r \frac{d}{d r} \phi_{m, n}\right)-k_{\perp}^{2} f\left(\omega, k_{\|}\right) \phi_{m, n}=g\left(k_{\|}\right) \phi_{m, n}
$$

where

$$
f\left(\omega, k_{\|}\right)=\left(\omega / v_{A}\right)^{2}-k_{\|}^{2}-\alpha\left(\omega-k_{\|} v_{b}\right),
$$

$k_{\perp}^{2}=(m / r)^{2}+(n / R)^{2}$, and $g\left(k_{\|}\right) \approx\left(d^{2} k_{\|} / d r^{2}\right) k_{\|}$. The Alfvén resonance coefficient $f\left(\omega, k_{\|}\right)$ is compared with the local dispersion relation (1). Here $\ddot{k}_{\|}$is a function of $r$.

Let $r=r_{0}$ be inside the plasma, and $k_{\|}\left(r_{0}\right)=k_{0}$. We approximate

$$
k_{\|}(r)=k_{0}+\beta x
$$

where $x=r-r_{0}$. Then,

$$
\begin{aligned}
f\left(\omega, k_{\|}(r)\right) & =\Delta^{2}-\beta^{2} y^{2}, \\
\Delta^{2} & =\left(\omega / v_{A}\right)^{2}-\alpha \omega+\alpha^{2} v_{b}^{2} / 4 \\
y & =x+\beta^{-1}\left(k_{0}-\alpha v_{b} / 2\right) .
\end{aligned}
$$

Here we assume that $y=0$ occurs in the plasma. Since $y^{2} \approx \beta^{-2}\left(k_{\|}-\alpha v_{b} / 2\right)^{2}$, and since $\left|k_{\|}\right|$is lower bounded because of the toroidal periodicity, this assumption implies that $\left|\alpha v_{b}\right|$ is sufficiently large [See the local dispersion relation (2)]. For small $x,(12)$ is approximated by

$$
\frac{d}{d y}\left[\left(\Delta^{2}-\beta^{2} y^{2}\right) \frac{d}{d y} \phi_{m, n}\right]-k_{\perp}^{2}\left(\Delta^{2}-\beta^{2} y^{2}\right) \phi_{m, n}=g_{0} \phi_{m, n}
$$


where $g_{0}=g\left(k_{0}\right)$.

Using the method of, ${ }^{7}$ we convert (13) into a Schrödinger-type equation. The Fourier transform of $(13)\left[y \leftrightarrow \eta, \phi_{m, n}(y) \leftrightarrow \hat{\phi}_{m, n}(\eta)\right]$ yields the equation obeyed by $\hat{\phi}_{m, n}(\eta)$

$$
\frac{d}{d \eta}\left[\left(\eta^{2}+k_{\perp}^{2}\right) \frac{d}{d \eta} \widehat{\phi}_{m, n}\right]+\frac{\Delta^{2}}{\beta^{2}}\left(\eta^{2}+k_{\perp}^{2}\right) \widehat{\phi}_{m, n}=\frac{g_{0}}{\beta^{2}} \widehat{\phi}_{m, n} .
$$

Defining $\xi=\eta / k_{\perp}, \epsilon=k_{\perp}^{2} \Delta^{2} / \beta^{2}, \bar{g}_{0}=g_{0} / \beta^{2}$, and $\psi_{m, n}=\left(1+\xi^{2}\right)^{1 / 2} \widehat{\phi}_{m, n},(14)$ becomes

$$
\left(-\frac{d^{2}}{d \xi^{2}}+V(\xi)\right) \psi_{m, n}=\epsilon \psi_{m, n}
$$

where $\epsilon$ is the eigenvalue and the effective potential is given by

$$
V(\xi)=\frac{1}{\left(1+\xi^{2}\right)^{2}}+\frac{\bar{g}_{0}}{1+\xi^{2}} .
$$

We obtain the frequency of the Alfvén wave by $\Delta^{2}=\epsilon \beta^{2} / k_{\perp}^{2}$, which now reads

$$
\frac{\omega}{\omega_{c i}}=\frac{\delta}{2}\left[1 \pm \sqrt{1-\left(\frac{v_{b}}{v_{A}}\right)^{2}+\epsilon\left(\frac{2 \beta v_{A}}{\delta \omega_{c i} k_{\perp}}\right)^{2}}\right] .
$$

This result for nonuniform $\mathbf{B}_{0}$ can be compared with the local dispersion relation (2).

From (15) and (16) we easily understand the spectral structure of the Alfvén waves. The spectrum $\epsilon$ is real, and the continuous spectrum spans $\epsilon \geq 0$. Solutions may also exist for $\epsilon<$ 0 , if the potential $V(\xi)$ has a sufficiently deep negative dip, which is given by the second term of (16) if $\bar{g}_{0}<0$ [Fig. 1]. To obtain a point spectrum, we need $\bar{g}_{0}<-1 / 4 .^{7}$ The eigenfunction corresponding to a negative point spectrum represents a nonsingular eigenmode, which is free from heavy continuum damping.

A significance of the mode with $\epsilon<0$ is that it can be excited by a sub-Alfvénic $\left(v_{b}<v_{A}\right)$ beam [Eq. (17)]. A part of the free energy to excite such a wave comes from the twist of $\mathbf{B}_{0}$ given by the bulk component of the current. By using a simple model, we now show that the background helicity yields negative $\bar{g}_{0}$. Consider a slab plasma with Cartesian components of the magnetic field given by

$$
\mathbf{B}_{0}=B_{0}\left(0, \sin \lambda\left(x+x_{0}\right), \cos \lambda\left(x+x_{0}\right)\right),
$$


where $x_{0}$ is a constant and $\lambda$ is proportional to the background helicity [See (4) and (5)]. Consider a wave such that $\phi_{m, n} \exp i(-m y+n z-\omega t)$, where $y$ and $z$ parallel the poloidal and toroidal coordinates, respectively. We obtain

$$
\bar{g}_{0}=-\frac{\left(n \cos \lambda x_{0}-m \sin \lambda x_{0}\right)^{2}}{\left(n \sin \lambda x_{0}+m \cos \lambda x_{0}\right)^{2}} .
$$

In Fig. 2 we pot $\bar{g}_{0}$ as a function of $\lambda$. A large helicity, which is proportional to $\lambda$, yields a large negative $\bar{g}_{0}$.

W - have shown that the mean-field helicity associated with the electron beam decays into the helicity associated with the left-hand circularly polarized Alfvén wave. In addition, we have shown that the background helicity due to the bulk plasma current also contributes to the wave excitation, the threshold velocity of the beam electrons at which the Cherenkov emission occurs is reduced below the Alfvén speed, and it becomes possible for a sub-Alfvénic electron beam to excite a nonsingular eigenmode.

We note that the Alfvén wave instability resembles the kink-mode instability of the electron beam. ${ }^{4}$ The magnetohydrodynamic (MHD) kink modes also modify the background helicity, while they do not produce wave helicity. The ideal MHD is described by an Hermitian operator, and hence a complex frequency describing a wave instability does not occur. The dissipative MHD system allows damped waves, and the natural direction of helicity transport is from the waves to the mean field. In the present model, the free energy of the streaming electrons induces the wave helicity.

The work was supported by the U.S. Department of Energy contract \#DE-FG05-80ET53088 .

One of the authors (Z.Y.) is grateful to Profs. J.A. Tataronis, S.C. Prager, and Dr. J.S. Sarff for discussions during his stay at The University of Wisconsin. Travel for Z.Y. was supported by the Joint Institute of Fusion Theory. 


\section{References}

${ }^{1}$ R.R. Mett and J.A. Tataronis, Phys. Rev. Lett. 63, 1380 (1989).

${ }^{2}$ J.B. Taylor, Phys. Rev. Lett. 63, 1384 (1989).

${ }^{3}$ In a high temperature plasma, the electron thermal speed is much higher than the Alfvén speed. A relatively strong toroidal induction electric field can easily generate an appreciable amount of slide-away electrons carrying a large current. Hence the assumption of an electron beam may apply. For a direct observation of fast electron stream, see Y. Yagi, Y. Hirano, T. Shimada, K. Hattori, Y. Maejima, I. Hirota, and A.A. Newton, Plasma Phys. Controlled Fusion 33, 1391 (1991).

${ }^{4}$ A. Hasegawa, Plasma Instabilities and Nonlinear Effects (Springer-Verlag, Berlin, 1975), sec. $2.2 \mathrm{~B}$.

${ }^{5}$ The present mechanism of wave production may apply to a reversed-field pinch experiment. ${ }^{3}$ In W. Shen, R. N. Dexter, and S.C. Prager, Phys. Rev. Lett. 68, 1319 (1992), strongly anisotropic magnetic fluctuations are shown in the range of $\omega>10^{5} \mathrm{~Hz}$, which are close to the local resonances $\left(\left|k_{\|}\right| \ll\left|k_{\perp}\right|\right)$. Substituting the measured $k_{\|}\left(\approx 0.7 m^{-1}\right)$ into the dispersion relation with $v_{A} \approx 10^{6} \mathrm{~m} / \mathrm{sec}$, we obtain $\omega$ in the relevant range. The phase propagates in the preferential direction opposite to the mean current.

${ }^{6}$ The reciprocal response of the beam to the emission of the wave may be calculated by the helicity and energy conservation laws. Since the present instability is of the negative energy type, we invoke the total energy conservation. The whistler wave modulates the beam to diminish the energy, as well as the helicity because it is $R$-polarized.

${ }^{7}$ S.M. Mahajan, Phys. Fluids 27, 2238 (1984). 


\section{Figure Captions}

1. The effective potential $V(\xi)$ with $\bar{g}_{0}=0,-0.5,-1$, and -1.5 . With a deep enough potential dip, a localized (nonsingular) eigenmode occurs, which has a negative eigenvalue $\epsilon$.

2. The potential parameter $\bar{g}_{0}$ as a function of $\lambda$. Here $n / m=0.1$ and $x_{0}=\pi / 10$. 


\section{FIGLRES}

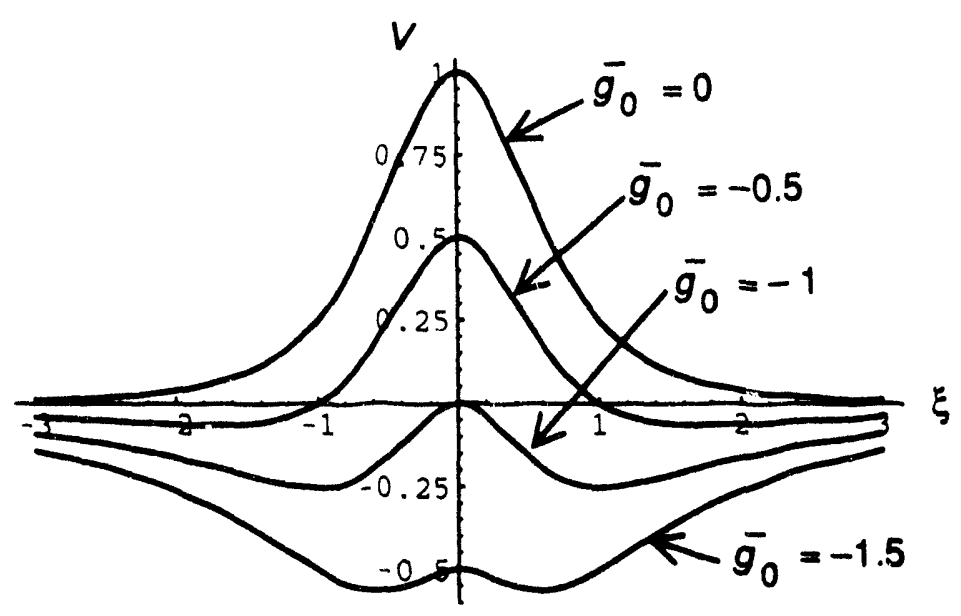

FIG. 1. The effective potential $V(\xi)$ with $\overline{g_{0}}=0,-0.5,-1$, and -1.5 . With a deep enough potential dip. a localized (nonsingular) eigenmode occurs, which has a negative eigenvalue $\epsilon$.

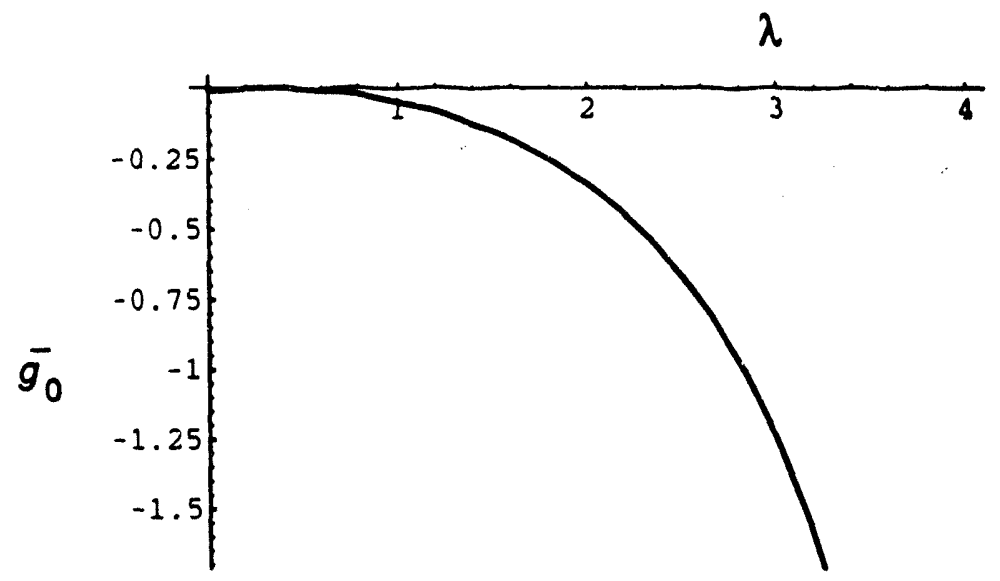

FIG. 2. The potential parameter $\overline{g_{0}}$ as a function of $\lambda$. Here $n / m=0.1$ and $x_{0}=\pi / 10$. 
11
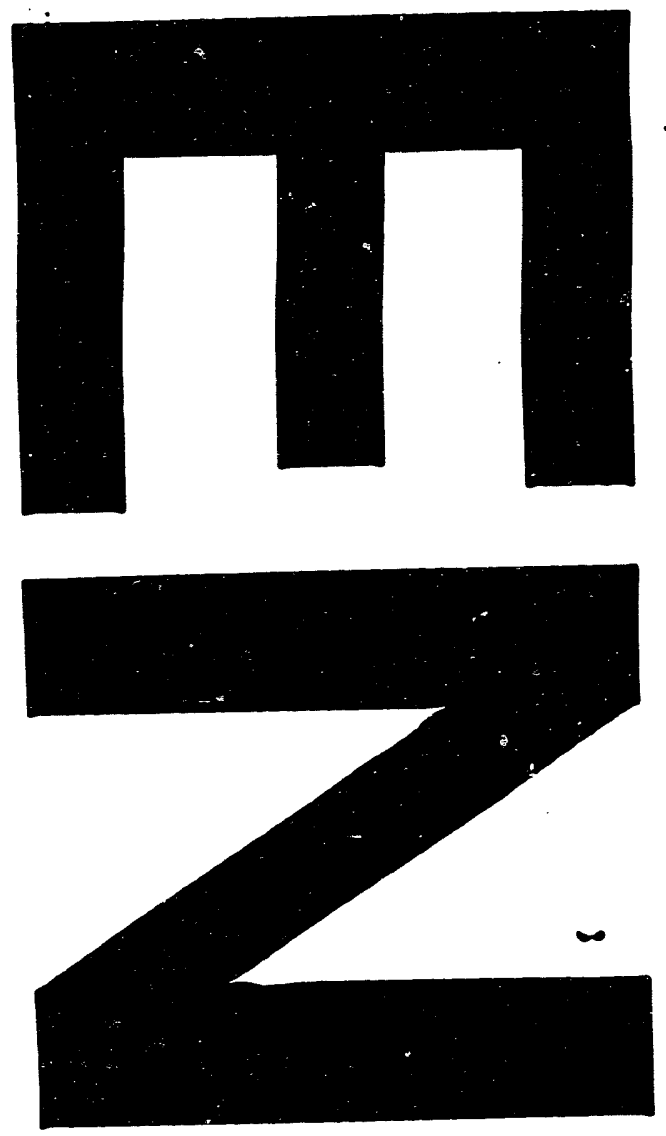

$G \pi$
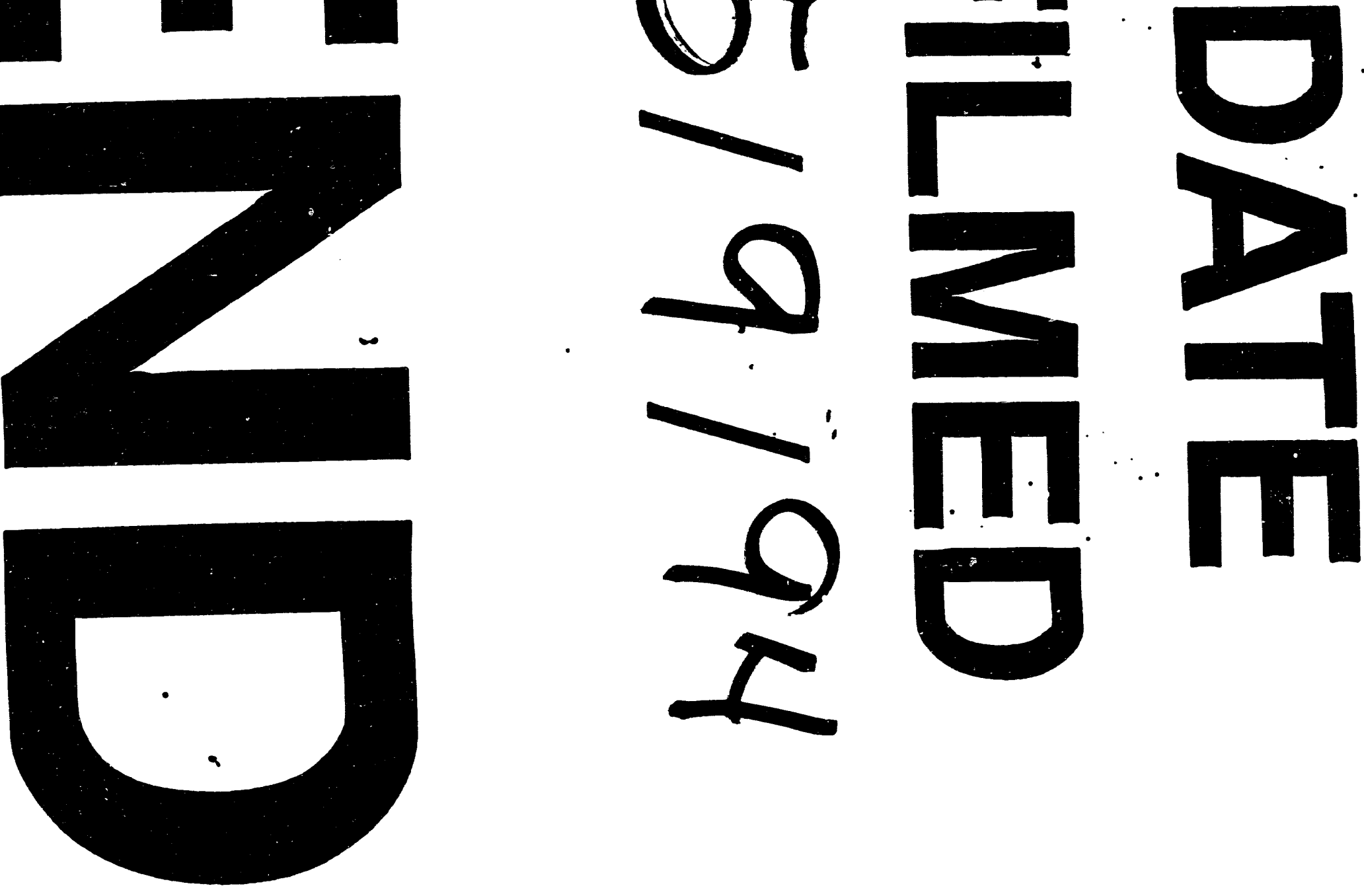
